\title{
Olfactory classical conditioning in neonatal mouse pups using thermal stimuli
}

\author{
Bieke Bollen $^{\mathrm{a}, \mathrm{b}, *}$, Boris Matrot $^{\mathrm{b}, \mathrm{c}}$, Nelina Ramanantsoa ${ }^{\mathrm{b}, \mathrm{c}}$, Omer Van den Bergh $^{\mathrm{d}}$, Rudi D’Hooge ${ }^{\mathrm{a}}$, \\ Jorge Gallego ${ }^{\mathrm{b}, \mathrm{c}}$ \\ a Laboratory of Biological Psychology, University of Leuven, Belgium \\ b INSERM, UMR676, Robert Debré Hospital, Paris, France \\ c University Denis Diderot, Faculty of Medicine, Paris, France \\ ${ }^{\mathrm{d}}$ Research Group on Health Psychology, University of Leuven, Belgium
}

\section{A R T I C L E I N F O}

\section{Article history:}

Received 7 September 2011

Received in revised form

29 November 2011

Accepted 19 December 2011

Available online 12 January 2012

\section{Keywords:}

Neonatal

Mice

Phenotyping

Thermotaxis

Conditioning

Odor preference

\begin{abstract}
A B S T R A C T
Mouse models are increasingly used to investigate genetic contributions to developmental disorders in children, especially newborns. In particular, early cognitive assessment in newborn mice is critical to evaluate pediatric drug efficacy and toxicity. Unfortunately, methods for behavioral tests in newborn mice are scarce. Therefore, developing such tests for newborn mice is a priority challenge for neurogenetics and pharmacological research. The aim of the present study was to develop a conditioning method well suited to high-throughput cognitive screening in newborn mice. To this end, we developed an odorpreference conditioning test using ambient temperature as an unconditioned stimulus (US) and artificial odors as conditioned stimuli (CS). First, we showed that mouse pups move toward the thermoneutral temperature when offered a choice between a thermoneutral and cold environment, thus showing thermotaxis. Second, we conducted a classical conditioning paradigm in pups aged six to ten days. In terms of central nervous system development, this period corresponds to extreme prematurity to early postterm period in humans. During acquisition, the pups were alternatively exposed to odor CS paired with either cold or warm temperatures. Immediately after acquisition, the pups underwent a two-odor choice test, which showed preference for the odor previously paired with the warm temperature, thus showing conditioning. The proposed paradigm is easy to conduct, and requires modest experimenter interference. The method is well suited for high-throughput screening of early associative disorders in newborn mice.
\end{abstract}

(c) 2012 Elsevier B.V. All rights reserved.

\section{Introduction}

As mice are the preferred mammalian species for genetic studies, methods for behavioral phenotyping are important instruments to investigate the functional significance of specific genes and their role in cognitive disorders [1]. The development of methods for assessing behavior in adult mice has received considerable attention [2], in sharp contrast to methods for behavioral assessment in developing, especially newborn mice. Unfortunately, the paucity of methodological tools suitable for newborn mice hampers the identification of early predictors of adult learning and memory deficits [3]. Furthermore, mice are increasingly used for modeling and investigating neurodevelopmental disorders, especially autism [4], in which early cognitive assessment is clinically relevant [5]. In

\footnotetext{
* Corresponding author at: Laboratory of Biological Psychology, University of Leuven, Tiensestraat 102, box 3714, 3000 Leuven, Belgium. Tel.: +32 16 326010; fax: +32 16326099 .

E-mail address: bieke.bollen@psy.kuleuven.be (B. Bollen).
}

addition, as emphasized by regulatory agencies ${ }^{1}$, early behavioral assessment is mandatory to evaluate pediatric drug efficacy and neurotoxicity, because of differences of receptor expressions and functions between immature and mature organisms. For all these reasons, developing cognitive tests for newborn mice is a priority challenge for neurogenetics and pharmacological research.

The aim of the present study was to develop a simple, easy to standardize classical conditioning protocol suitable for highthroughput screening of associative abilities in newborn mice. Previous studies showed that pairing artificial odors (conditioned stimuli, (CS)) and stroking with a brush (unconditioned stimulus, as a surrogate of maternal licking and grooming) induced an effective conditioned odor preference in newborn mice [6]. This conditioning method proved efficient to show learning deficits in newborn mice with brain lesions similar to that of preterm infants,

\footnotetext{
${ }^{1}$ Food and Drug Administration (FDA): Nonclinical Safety Evaluation of Pediatric Drug Products; http://www.fda.gov/cder/guidance/5671fnl.htm; European Medical Agency (EMA): Guideline on the need for non-clinical testing in juvenile animals of pharmaceuticals for pediatric indications, http://www.emea.europa.eu.
} 
and to assess functional efficacy of experimental neuroprotective treatments $[7,8]$. However, odor-stroke conditioning is difficult to standardize, due to strong experimenter effects on stroking, which prompted us to broaden the range of available conditioning methods in newborn mice.

Here, we describe an original conditioning method, using ambient temperature as an unconditioned stimulus (US), which is easy to implement and which overcomes the experimenter-related problem inherent to odor-stroke conditioning. We hypothesized that mice, like most altricial animals, show very limited thermoregulatory capacities during early postnatal development, and that they mainly rely on behavioral strategies, such as huddling and thermotaxis to maintain body temperature [9-12]. This being confirmed, we hypothesized that newborn mice would be able to associate artificial odors with ambient temperature, in keeping with their proven ability to associate odors with biologically relevant stimuli, such as stroking [6] or lack of oxygen [13]. Experiments were conducted in neonatal mice of 3-10 days of postnatal age. Compared with human infants, this period roughly corresponds to very preterm (about 25 weeks of gestational age) to term infants [14].

\section{Material and methods}

\subsection{Animals}

In the thermotaxis $(N=28)$, the first conditioning study $(N=64)$, and extinction study $(N=15)$, mouse pups were obtained from 16 C57BL6 female mice (Janvier, Le Genest-St-Isle, France) housed at $24^{\circ} \mathrm{C}$ with a $12 \mathrm{~h} / 12 \mathrm{~h}$ light/dark cycle and fed ad libitum. Sixteen litters were used (mean number of pups per litter: $6.7 \pm 2.2$ ). Pups were tested at postnatal ages P3 $(n=12)$, P6 $(n=38)$, P7 $(n=20)$, P8 $(n=3)$, P9 $(n=24)$ and P10 $(n=10)$ (P0, the first day of life), with mean weight per age $1.9 \pm 0.2$, $3.7 \pm 0.5,3.6 \pm 0.6,3.7 \pm 0.1,4.9 \pm 0.6$, and $5.2 \pm 0.4 \mathrm{~g}$, respectively.

In the second conditioning study (replication), mouse pups were obtained from eight Swiss female mice (Janvier, Le Genest-St-Isle, France) housed at $24^{\circ} \mathrm{C}$ with a $12 \mathrm{~h} / 12 \mathrm{~h}$ light/dark cycle and fed ad libitum. Eight litters were used (mean number of pups per litter: $13.3 \pm 4.4)$. Pups were tested at postnatal ages $\mathrm{P} 5(n=22), \mathrm{P} 6(n=8)$, P7 $(n=79)$ and P8 $(n=8)$, with mean weight per age $3.8 \pm 0.2,4.2 \pm 0.3,3.6 \pm 1.0$, and $5.3 \pm 0.4 \mathrm{~g}$, respectively.

Experiments were carried out in accordance with the European Communities Council Directive of November 1986 (86/609/EEC) regarding the care and use of animals for experimental procedures, in compliance with the regulations of the Ministère de l'Agriculture et de la Forêt, Service Vétérinaire de la Santé et de la Protection Animale (permission \# A 94-028-21). Experimental protocols were approved by the Animal Experiments Committee of the University of Leuven and the Institut National de la Santé et de la Recherche Médicale (INSERM) Institutional Review Committee.

\subsection{Acquisition setup}

Acquisition took place in a custom apparatus (Fig. 1A) composed of two cylindershaped recipients filled with water. The water was thermoregulated to maintain the temperature at either $26^{\circ} \mathrm{C}$ or $33^{\circ} \mathrm{C}$. Each recipient contained a cylindrical metal chamber that was immersed in the water, and equipped with a metallic grid floor, which was secured $2 \mathrm{~cm}$ above the bottom of the chamber. An odor-containing cotton wad placed in an aluminum foil dish was positioned underneath the grid. This system allowed exposing the pups to paired odor and thermal stimuli.

\subsection{Test setup}

The test apparatus was a place preference system previously described [6-8,13]. Briefly, this apparatus (Fig. 1B) was composed of two identical Plexiglas boxes separated by a Plexiglas beam, which delimited a neutral zone. Each box contained an aluminum dish with an odor-containing cotton wad. The two boxes and neutral zone were covered by a $13 \mathrm{~cm} \times 20 \mathrm{~cm}$ metallic mesh floor $(1.5 \mathrm{~mm} \times 3.0 \mathrm{~mm}$ meshwork). Temperature on the mesh was maintained at $24^{\circ} \mathrm{C}$.

\subsection{Conditioned stimuli}

Banana and peppermint odor were chosen on the basis of a spontaneous odor preference test in a small number of mice (data not shown), during which no preference for one of these odors was observed. We used $0.5 \mathrm{ml}$ of peppermint odor $(97 \%$ menthyl acetate; Aldrich, Steinheim, Germany) and $0.25 \mathrm{ml}$ of banana odor ( $97 \%$ Butyl propionate; Aldrich, Steinheim, Germany).

\subsection{Procedure}

Acquisition trials consisted in placing the pup over the CS+ odor, i.e. the odor associated with warmth $\left(33^{\circ} \mathrm{C}\right.$, this temperature is considered to be close to thermoneutrality in newborn rodents $[15,16])$, during $90 \mathrm{~s}$ (s) and over the CS- odor, associated to cold $\left(26^{\circ} \mathrm{C}\right)$, also for $90 \mathrm{~s}$. Thermographic studies in newborn rats indicate that $26^{\circ} \mathrm{C}$ corresponds to maximal thermogenesis by brown adipose tissue (a thermogenic organ located in the interscapular region) and can therefore be considered cold [16]. Pups were not handled during this 90 s period. Acquisition consisted of three CS+/US presentations alternating with three CS- presentations. To control for initial differences in preference for the two odors, banana was the CS+ and peppermint the CS- in half the pups (the banana CS+ group) and vice versa in the other half (the peppermint CS+ group). The pups were randomly extracted from the litter one by one and were assigned to the banana CS+ group or the peppermint CS+ group. The first acquisition trial was a CS+ trial in half the pups and a CS- in the other half.

Immediately after acquisition, the mouse pup was placed in the test apparatus and subjected to five successive tests for conditioning (each test lasted $60 \mathrm{~s}$ ). During each test, the pup was placed on the neutral zone (along the axis of this zone) and the experimenter measured the time spent over each odor. After each one-minute test trial, the pup was removed from the apparatus and immediately positioned for the following test trial. The temperature in the test apparatus was maintained at $24^{\circ} \mathrm{C}$. A cold temperature was chosen to motivate the pups to search for warmth. A pup was considered to prefer an odor when it moved at least its snout toward that odor beyond the edge of the neutral zone. Thus, a movement of the head was sufficient to assess odor preference. As soon as a pup's snout crossed one of the edges of the neutral zone, a timer was started to measure the time spent over the CS. In practice, positioning of the snout over an odor zone was systematically accompanied by movements of the head, the shoulders and the anterior paws. The starting position during the first test was counterbalanced across animals and the direction in which the pup was placed on the neutral line was changed (i.e., $180^{\circ}$ rotation) at the beginning of each subsequent test. The mesh was carefully wiped between testing. The position of the odors was not changed in order to avoid mixing the odors. The experimenter also used different gloves for manipulating odors and for handling mouse pups to avert possible confounds. The acquisition setup was carefully brought to the correct temperature before acquisition trials started and was also rigorously checked during acquisition trials. Only minor deviations of $0.5^{\circ} \mathrm{C}$ to the postulated temperatures of $26^{\circ} \mathrm{C}$ and $33^{\circ} \mathrm{C}$ were allowed.

Body temperature (by means of a thermocouple probe placed at the level of the interscapular region) and weight of the mouse pup were measured before acquisition. Acquisition and test trials were conducted by the same experimenter. The behavior of the pups during the test was recorded using a webcam and scored by an experimenter that was blind for the experimental group.

\subsection{Thermotaxis}

Thermotaxis was assessed by measuring the pups' place preference when placed in a choice situation between cold and thermoneutral environments [10]. The test apparatus described earlier (Fig. 1B) contained no odors during the test for thermotaxis. Instead, the two small boxes were filled with water. The temperature of the water was adjusted so that on one side of the test apparatus the temperature just above the grid was approximately $33^{\circ} \mathrm{C}$, while on the other side of the test apparatus it was around $26^{\circ} \mathrm{C}$ (only small deviations of $0.5^{\circ} \mathrm{C}$ were allowed). Preference for one or other side was assessed as described above for the conditioned odor preference test.

\subsection{Replication}

Because behavioral tests in adult mice are strongly influenced, by genetic background, housing and testing conditions [17], we examined whether neonatal thermal conditioning may operate in different experimental conditions and in a different mouse strain. Conditioning tests were conducted in a second center, using a modified version of the acquisition setup using thermoregulated water and a different presentation of odors (i.e. around, rather than below the pup). In this way, the pup was placed on a metal floor (compared to a grid in the first experiment) and thus in more direct contact with the thermal stimuli. Also, in an attempt to strengthen conditioning, we slightly modified the protocol by increasing the number of trials: the duration of each acquisition trial was $45 \mathrm{~s}$ (instead of $90 \mathrm{~s}$ ). In this way, the number of exposures to the CS+ and CS - was doubled (six CS+/US-CS- alternations instead of three), without prolonging acquisition ( $9 \mathrm{~min}$ ). The conditioning protocol was replicated using two odor pairs (banana-peppermint and banana-lemon). These odor pairs were chosen on the basis of spontaneous preference experiments in Swiss mouse pups (data not shown): $1.5 \mathrm{ml}$ peppermint ( $97 \%$ menthyl acetate; Aldrich, Steinheim, Germany), $0.5 \mathrm{ml}$ of banana (97\% Butyl propionate; Aldrich) and $1 \mathrm{ml}$ of lemon odor (97\% Limonene, Aldrich).

\subsection{Statistical analysis}

The time spent on each odor, was subjected to analysis of variance (Repeated Measures ANOVA) with CS (CS+ versus CS-) and test number (5 levels) as the within-subject factors and group (CS+ banana versus CS+ peppermint) as the 
(A) $26^{\circ} \mathrm{C}$

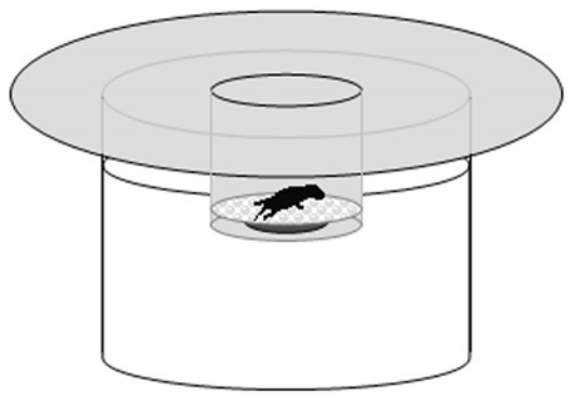

$33^{\circ} \mathrm{C}$

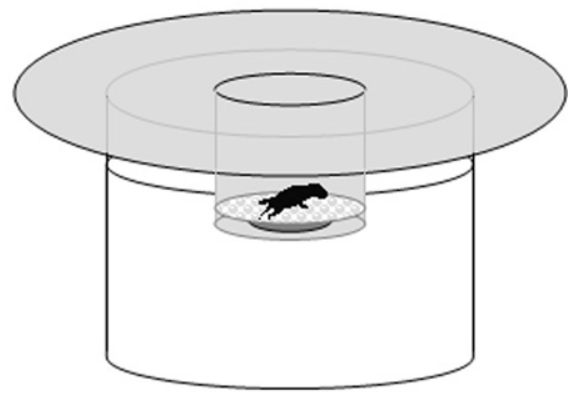

(B)

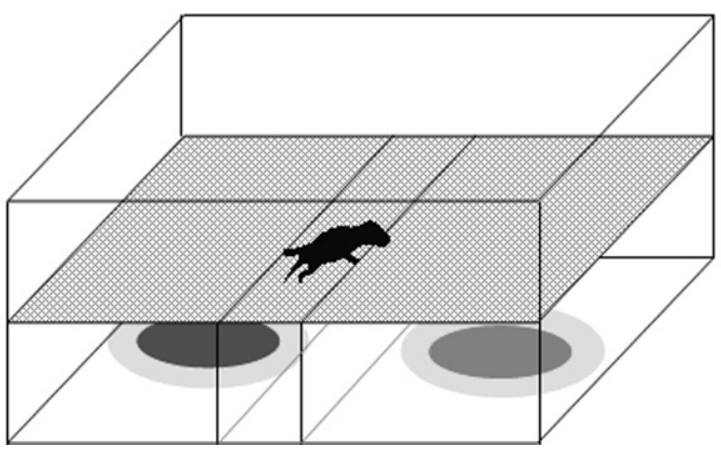

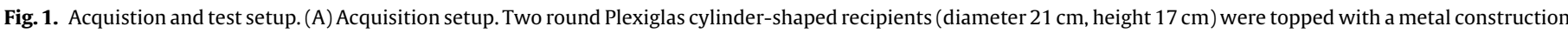

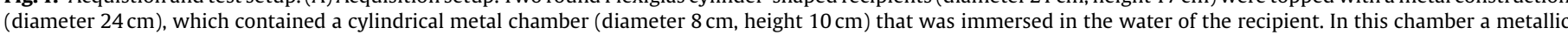

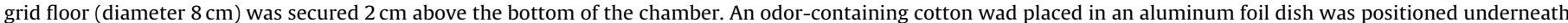

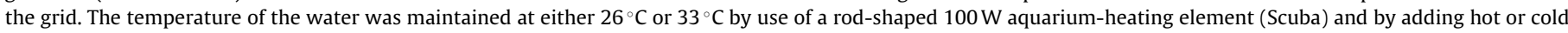

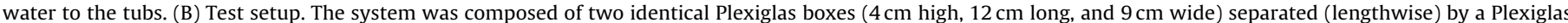

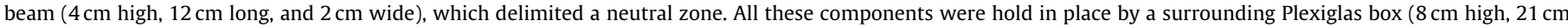

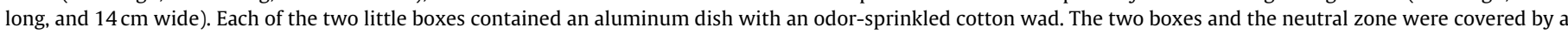
$13 \mathrm{~cm} \times 20 \mathrm{~cm}$ metallic mesh floor $(1.5 \mathrm{~mm} \times 3.0 \mathrm{~mm}$ meshwork $)$ and placed in a hood.

between-subject factors (Statview 5, Abacus Concepts, Berkeley, CA). Figures were created using GraphPad Prism 5 (GraphPad Software Inc., San Diego, California, USA). In experiments where different age groups were included, age was also included as a between-subjects factor. Litter had no significant effect, neither as a main between-factor nor in interaction with other factors, and will not be mentioned further.

\section{Results}

\subsection{Thermotaxis}

We investigated thermotaxis in 28 mouse pups from 3 litters at P3 $(n=12)$, P6 $(n=11)$, and P9 $(n=5)$. Mouse pups spent significantly more time on the thermoneutral side of the grid $\left(33^{\circ} \mathrm{C}\right)$ than over the cold side $\left(26^{\circ} \mathrm{C}\right.$, main effect of temperature: $F_{1,25}=21.56$, $p<0.0001$, Fig. 2A). This preference did not significantly depend on age (temperature-by-age interaction: $F_{2,25}=1.12, p=0.343$, Fig. $2 \mathrm{~B}$ ). Mouse pups in general spent more time outside of the neutral zone with increasing age (non-significant trend: main effect of age: $\left.F_{2,25}=2.28, p=0.124\right)$. The results show that, at an early age, mouse pups are able to discriminate between different ambient temperatures and are capable of moving toward the temperature that is physiologically most appropriate.

\subsection{Thermal conditioning}

Then, we examined whether mouse pups were able to learn a preference for an odor previously paired with a thermoneutral temperature over an odor paired with a cold temperature.
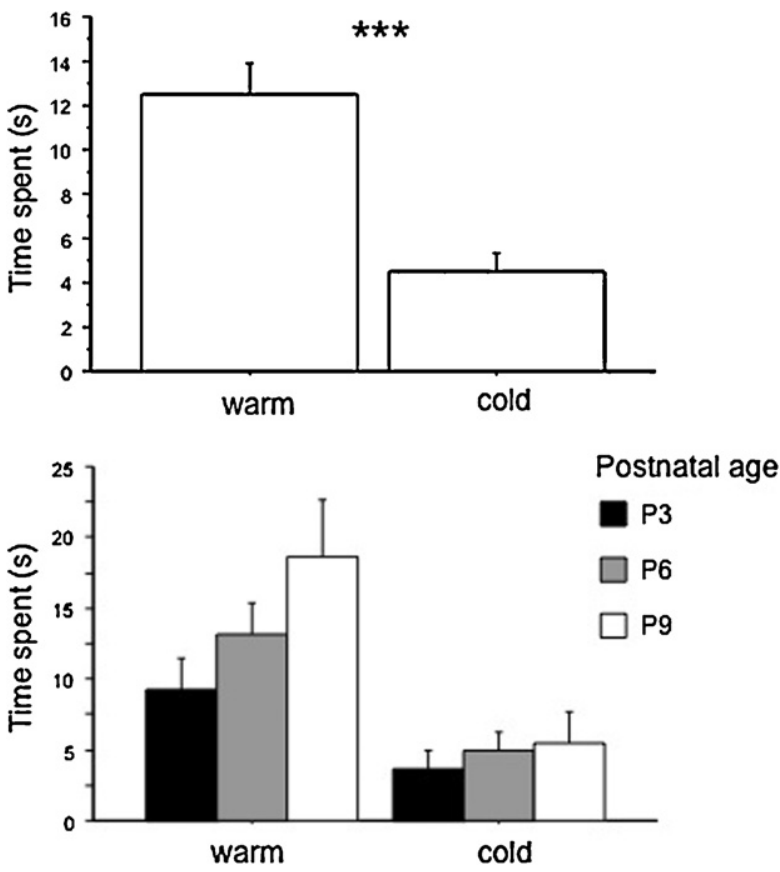

Fig. 2. Thermotaxis in neonatal mouse pups. (A) Main effect of temperature. All age groups (P3, P6, P9) were pooled together. (B) Age-by-temperature interaction effect. See text for statistical analyses. ${ }^{* * *} p<.0001$. Values are means \pm SEM. 

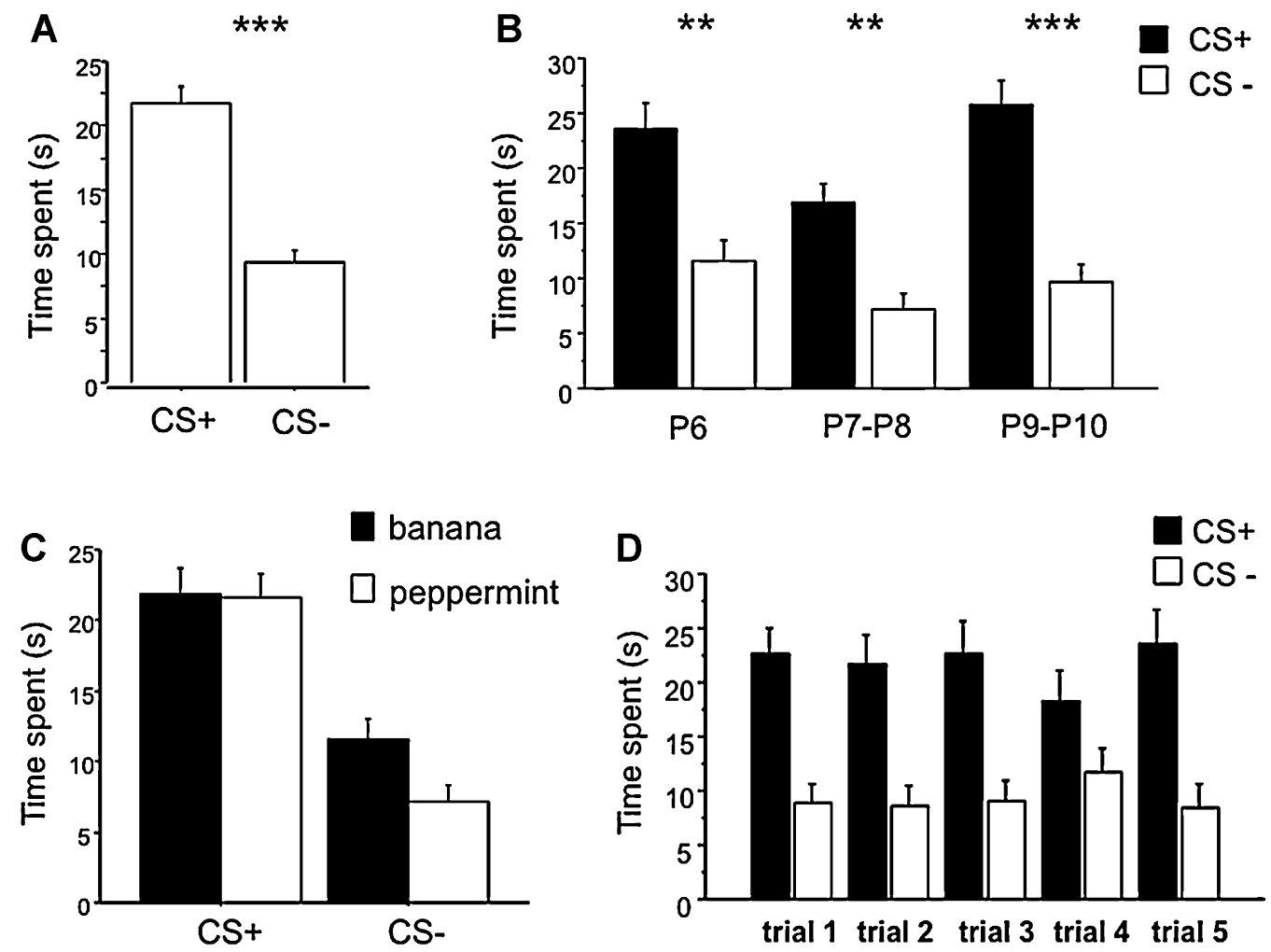

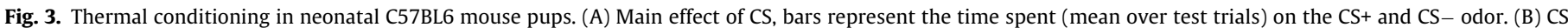

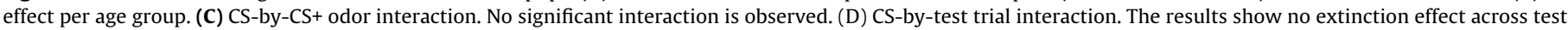
trials. See text for statistical analyses. ${ }^{* *} p<.001,{ }^{* * *} p<.0001$. Values are means \pm SEM.

Mouse pups ( $n=64)$ were tested immediately after acquisition at P6 $(n=19)$, P7-P8 $(n=24)$ and P9-P10 $(n=21)$. The results show a clear preference for the odor that was previously paired with the thermoneutral temperature (CS+) compared to the odor ( $\mathrm{CS}_{-}-$) paired with the cold temperature (main effect of CS: $F_{1,58}=41.532$, $p<0.0001$, Fig. 3A). The odor used as CS+ (banana or peppermint odor) did not significantly influence the conditioned preference (CS by CS+ odor interaction effect: $F_{1,58}=1.16, p=0.287$, Fig. $3 C$ ). Also, age did not significantly influence the conditioned preference (no significant CS by age group interaction $F_{1,58}=0.983, p=0.390$, Fig. 3B). The general activity, i.e. the time spent outside the neutral zone during the test, did differ significantly between age groups $\left(F_{2,58}=6.88, p=0.002\right)$. This finding is primarily due to less exploratory behavior in a few animals from the P7-P8 age group and does not invalidate our results since mouse pups from this age group, despite their lower level of exploration, show a preference for the CS+ odor over the CS- odor. We observed no extinction effect over the course of the five successive tests (CS-by-test trial interaction: $F_{4,232}=0.75, p=0.557$, Fig. 3D).

We investigated extinction in an exploratory study in a separate small group of animals (C57BL6, $N=15$, at P6 $(n=8)$ and P9 $(n=7)$ ), in which the preference test was repeated every hour for $4 \mathrm{~h}$. Separate analyses of the different test blocks indicated that pups showed a preference for the CS+ odor only immediately $\left(F_{1,13}=14.53\right.$, $p=0.002)$ and $1 \mathrm{~h}$ after acquisition $\left(F_{1,13}=7.66, p=0.016\right)$. During the subsequent 3 tests no conditioned preference was observed

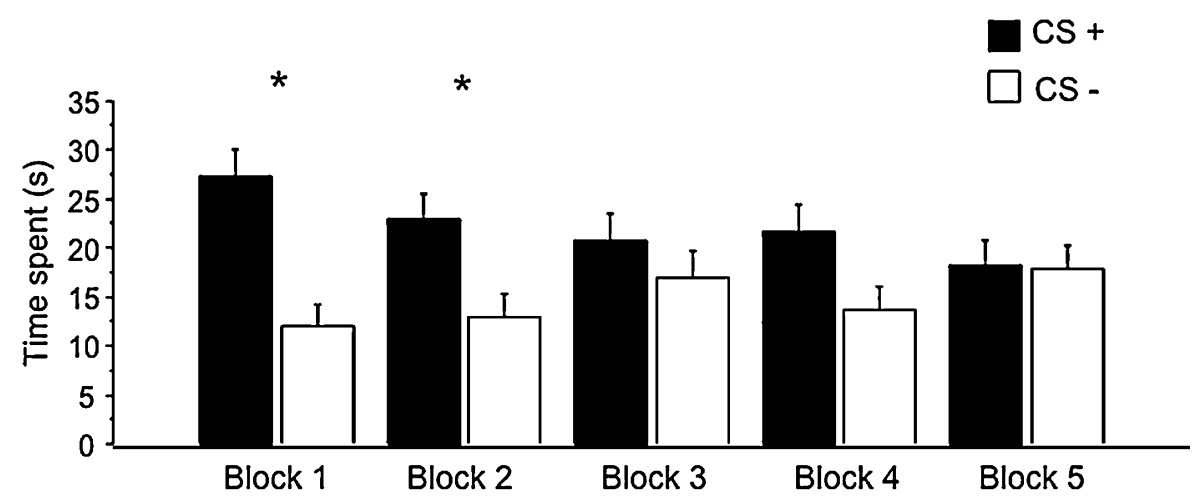

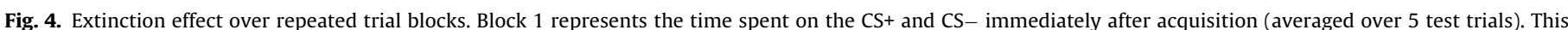

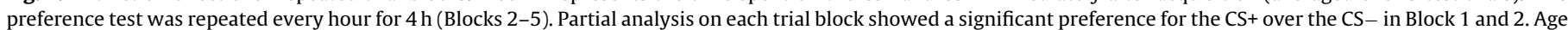

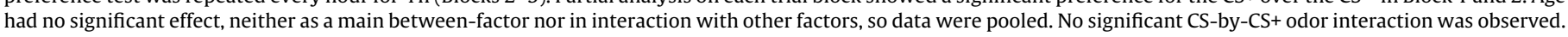
See text for statistical analyses. ${ }^{*} p<.05$. Values are means \pm SEM. 
A

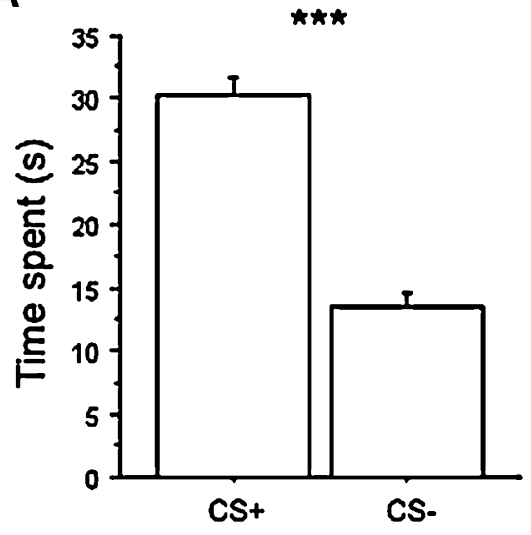

C

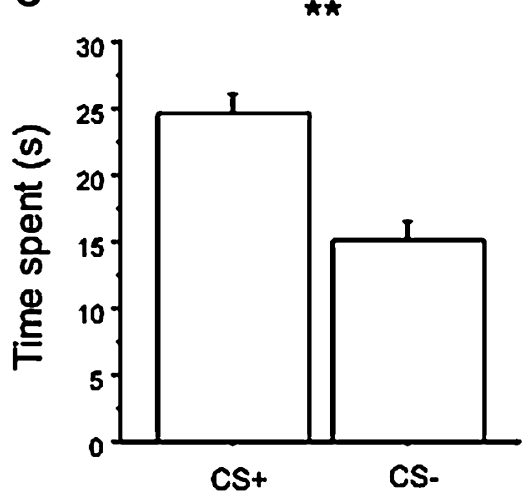

B
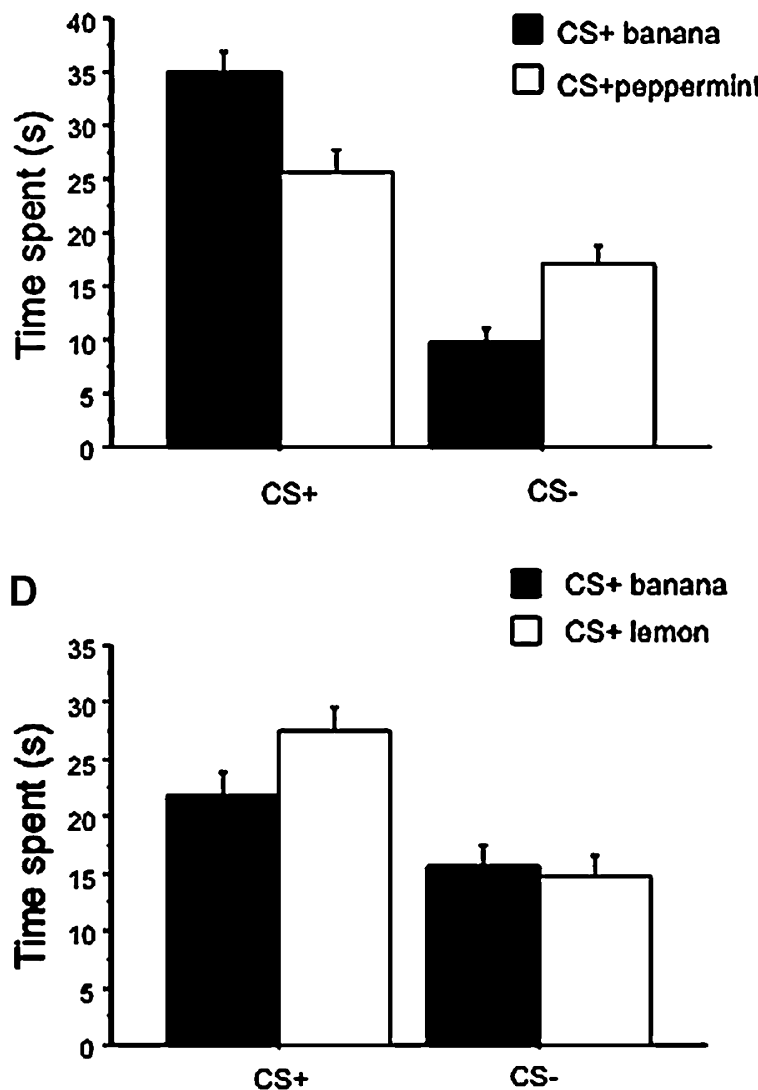

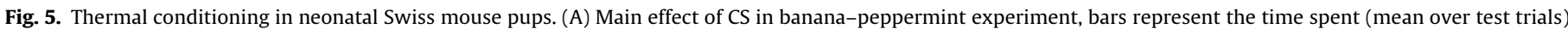

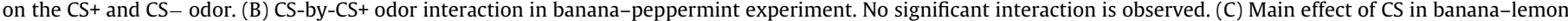

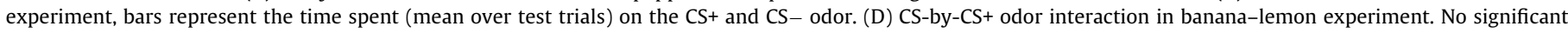
interaction is observed. ${ }^{* *} p<.001,{ }^{* * *} p<.0001$. Values are means \pm SEM.

$\left(F_{1,13}=0.44, p=0.52, F_{1,13}=1.75, p=0.209, F_{1,13}=0.00, p=0.988\right.$ respectively, Fig. 4$)$. These observations point toward an extinction effect, but the test block-by-CS interaction $\left(F_{4,52}=1.05, p>0.05\right)$ did not yield significance in this small sample of pups.

\subsection{Replication of conditioning in different setting}

The thermal conditioning protocol was applied by another experimenter in a different laboratory, using a modified protocol of the thermal conditioning paradigm in Swiss mouse pups instead of C57BL6 pups. Two different sets of odors were used as $\mathrm{CS}+/ \mathrm{CS}$ - pairs $(N=51$ (all aged P7) for banana-lemon and $N=55$ (at P5 $(n=22)$, P6 $(n=8)$, P7 $(n=28)$, and P8 $(n=8)$ ) for banana-peppermint conditioning). Also in this different setting, mouse pups spent significantly more time on the odor paired with the thermoneutral temperature (CS+) than on the odor associated with cold (banana-peppermint experiment: $F_{1,47}=26.15, p<0.0001$, Fig. 5A; banana-lemon experiment: $F_{1,49}=13.99, p=0.0005$, Fig. $5 C$ ). Again, no significant CS-by-CS+ odor interactions (banana-peppermint experiment: $F_{1,47}=2.29$, $p=0.094$, Fig. 5B; banana-lemon experiment: $F_{1,49}=1.65, p=0.205$, Fig. 5D) were found. In the banana-peppermint experiment, no main or interaction effect of age was observed (main effect of age: $F_{3,47}=1.17, p=0.333$; age-by-CS interaction: $F_{3,47}=1.88, p=0.147$ ).

\section{Discussion}

We took advantage of the thermotaxic behavior of newborn mice to design an associative learning test for neonatal mice, using artificial odors as conditioned stimuli and ambient temperature as the unconditioned stimulus. Neonatal mouse pups preferred the odor that had previously been paired with a thermoneutral ambient environment to an odor paired with a cold ambient temperature, hence showing conditioning. Thus, ambient temperature proved to serve as an adequate unconditioned stimulus for neonatal mouse pups in a classical conditioning learning test. The conditioning proved effective and reproducible from 5- to 10-day old pups.

The differential conditioning paradigm used in our study incorporated both within-subject (CS+ versus CS-) and within-odor controls (both banana and peppermint served as CS+ and CS-). This procedure allowed us to establish conditioned preference, separate from non-associative effects, such as a pre-existing preference for one of the two odors. A more detailed discussion on spontaneous odor preferences can be found elsewhere [6]. The lack of extinction across the five test trials done immediately after acquisition, as well as the presence of the conditioned preference after $1 \mathrm{~h}$, indicate that the conditioning effect is robust. The replication of the conditioned response in another experimental setting, on mice of a different strain, with a different acquisition setup and protocol, yielded similar results. This further underlines the robustness of the proposed learning protocol.

Our results show that mouse pups move toward the thermoneutral temperature when offered a choice between a thermoneutral and cold environment. These results extend the presence of thermotaxis in mouse pups, as previously described in rat $[10,18]$, rabbit [12] and hamster pups [11]. It is vital for mouse pups' and other altricial animals' survival to maintain an adequate body temperature. Because of their immature thermogenic capacities 
rodent pups rely heavily on behavioral strategies, such as huddling and thermotaxis, to maintain body temperature in absence of the dam $[9,11,19,20]$.

The main result of the present study was that mouse pups were able to associate artificial olfactory stimuli with ambient temperature. Conditioning was established after only three short acquisition trials, indicating a remarkable sensitivity of thermotaxic responses to learning processes. This sensitivity might reflect the biological significance of orienting toward the dam, the litter or in absence of both, toward a physiologically appropriate temperature, using other signaling (olfactory) cues. The present results indicate that warmth is a potent reinforcer in immature mammals. The present finding that neonates are able to associate odors with a temperature stimulus is a novel finding, not only in mice, but to our knowledge, also in other species. Recently, Kojima and Alberts [21] conducted several conditioning experiments using thermotactile stimuli and showed that warm ambient temperature alone did not induce a huddling preference for a conditioned odor in 15-day-old rat pups. However, a non-significant trend was observed, which suggested that warm ambience might be rewarding to younger, more thermally vulnerable pups. This possibility is supported by the present data that show effective conditioning in mouse pups aged P6-P10.

Other proposed methods of classical conditioning in rodent pups require important experimenter manipulation, for example stroking [6-8] or oral milk infusion in nutritive conditioning experiments in rat [22]. These methods are therefore poorly suited for high-throughput behavioral screening. Ambient temperature is a direct, easily controllable reinforcer, whereas the valence of stroking, which is thought to mimic maternal care, is difficult to control. Therefore, using ambient temperature as an unconditioned stimulus adds to the standardization of the conditioning protocol. Also, using ambient temperature as an unconditioned stimulus considerably reduces experimenter intervention during acquisition, which is the main bottleneck for increasing throughput. Exploratory experiments showed that preference scores during the test could be calculated automatically using a custom videotracking system, and that a single experimenter may run three pups simultaneously using this method.

The present test for conditioning, which was conducted immediately after acquisition, did not shed light on the long-term retention of the conditioned response. As a rule, retention of the conditioned responses is very short in newborn mammals [23], although longer retention was obtained using food reinforcers [23] or resumption of maternal care after separation [24]. Because the primary aim of our study was to design a conditioning protocol suitable for high-throughput screening, we focused on the shortterm effects of conditioning, which proved consistent and easy to observe. The results from the exploratory extinction study suggest a $1 \mathrm{~h}$ retention of the conditioned preference.

The present results did not clarify the neural substrate of odor/temperature conditioning. Moriceau and Sullivan [25] studied odor-shock conditioning in a rat attachment model and propose a specific neonatal odor learning circuitry during the first ten days of life, which involves the olfactory bulb, where learning is encoded, and the noradrenergic locus coeruleus. However, Bouslama et al. [7] showed that neonatal brain lesions caused by intracerebral ibotenate injection disrupt odor-stroke learning. Taken together, these results suggest that early postnatal conditioning based on pairing an odor with an US involves numerous and possibly widespread brain regions. The identification of these regions requires further investigation.

Cognitive phenotyping at early stages of mouse development has numerous applications, which concern the identification of genetic determinants of cognitive development, and also, as mentioned, the modeling of neurodevelopmental disorders.
High-throughput cognitive phenotyping is also mandatory to test possible adverse effects of candidate pediatric treatments on cognition at preclinical level. Of note, the early detection of cognitive disorders may be clinically relevant in neurological disorders of adulthood or aging, because early- but also late-onset neurological disorders might be in part born at early developmental stages [26].

\section{Conflict of interest}

The authors do not have any interests that might be interpreted as influencing the research that is described in the manuscript.

\section{Funding source}

This study was supported by Research Foundation Flanders (FWO) and Marguerite-Marie Delacroix Foundation (grants to Bieke Bollen).

\section{Acknowledgment}

We are greatly indebted to Aurélie Baudin, Thomas Vermeire, and Karolien Raeymaekers for excellent assistance in preliminary experiments.

\section{References}

[1] van der Staay FJ, Steckler T. Behavioural phenotyping of mouse mutants. Behav Brain Res 2001;125:3-12.

[2] Crawley JN. Behavioral phenotyping strategies for mutant mice. Neuron 2008;57:809-18.

[3] Akers KG, Nakazawa M, Romeo RD, Connor JA, McEwen BS, Tang AC. Early life modulators and predictors of adult synaptic plasticity. Eur J Neurosci 2006;24:547-54.

[4] Crawley JN. Mouse behavioral assays relevant to the symptoms of autism. Brain Pathol 2007;17:448-59.

[5] Branchi I, Campolongo P, Alleva E. Scopolamine effects on ultrasonic vocalization emission and behavior in the neonatal mouse. Behav Brain Res 2004;151:9-16.

[6] Bouslama M, Durand E, Chauviere L, Van den Bergh O, Gallego J. Olfactory classical conditioning in newborn mice. Behav Brain Res 2005;161:102-6.

[7] Bouslama M, Chauviere L, Fontaine RH, Matrot B, Gressens P, Gallego J. Treatment-induced prevention of learning deficits in newborn mice with brain lesions. Neuroscience 2006;141:795-801.

[8] Bouslama M, Renaud J, Olivier P, Fontaine RH, Matrot B, Gressens P, et al. Melatonin prevents learning disorders in brain-lesioned newborn mice. Neuroscience 2007; 150:712-9.

[9] Alberts JR. Huddling by rat pups: group behavioral mechanisms of temperature regulation and energy conservation. J Comp Physiol Psychol 1978;92: 231-45.

[10] Johanson IB. Thermotaxis in neonatal rat pups. Physiol Behav 1979;23:871-4.

[11] Leonard CM. Thermotaxis in golden hamster pups. J Comp Physiol Psychol 1974;86:458-69.

[12] Pacheco-Cobos L, Rosetti M, Distel H, Hudson R. To stay or not to stay: the contribution of tactile and thermal cues to coming to rest in newborn rabbits. J Comp Physiol A Neuroethol Sens Neural Behav Physiol 2003;189: 383-9.

[13] Bollen B, Bouslama M, Matrot B, D’Hooge R, Van den Bergh O, Gallego J. Learned defense response to hypoxia in newborn mice. Neurosci Lett 2007;420:268-72.

[14] Hagberg H, Peebles D, Mallard C. Models of white matter injury: comparison of infectious, hypoxic-ischemic, and excitotoxic insults. Ment Retard Dev Disabil Res Rev 2002;8:30-8.

[15] Renolleau S, Dauger S, Autret F, Vardon G, Gaultier C, Gallego J. Maturation of baseline breathing and of hypercapnic and hypoxic ventilatory responses in newborn mice. Am J Physiol Regul Integr Comp Physiol 2001;281:R1746-53.

[16] Blumberg MS, Sokoloff G. Thermoregulatory competence and behavioral expression in the young of altricial species - revisited. Dev Psychobiol 1998;33:107-23.

[17] van der Staay FJ. Animal models of behavioral dysfunctions: basic concepts and classifications, and an evaluation strategy. Brain Res Rev 2006;52:131-59.

[18] Kleitman N, Satinoff E. Thermoregulatory behavior in rat pups from birth to weaning. Physiol Behav 1982;29:537-41.

[19] Sokoloff G, Blumberg MS. Contributions of endothermy to huddling behavior in infant Norway rats (Rattus norvegicus) and Syrian golden hamsters (Mesocricetus auratus). J Comp Psychol 2002;116:240-6.

[20] Sokoloff G, Blumberg MS. Competition cooperation among huddling infant rats. Dev Psychobiol 2001;39:65-75. 
[21] Kojima S, Alberts JR. Warmth from skin-to-skin contact with mother is essential for the acquisition of filial huddling preference in preweanling rats. Dev Psychobiol 2011;53:813-27.

[22] Sullivan RM, Hall WG. Reinforcers in infancy: classical conditioning using stroking or intra-oral infusions of milk as UCS. Dev Psychobiol 1988;21:215-23.

[23] Cheslock SJ, Sanders SK, Spear NE. Learning during the newborn's first meal: special resistance to retroactive interference. Dev Sci 2004;7:581-98.
[24] Armstrong CM, DeVito LM, Cleland TA. One-trial associative odor learning in neonatal mice. Chem Senses 2006;31:343-9.

[25] Moriceau S, Sullivan RM. Unique neural circuitry for neonatal olfactory learning. J Neurosci 2004:24:1182-9.

[26] Ben-Ari Y. Neuro-archaeology: pre-symptomatic architecture and signature of neurological disorders. Trends Neurosci 2008;31:626-36. 